\title{
Physics of Celestial Scale Dumbbells
}

\author{
Leonardo Golubović ${ }^{1} \&$ Steven Knudsen ${ }^{1}$ \\ ${ }^{1}$ Physics and Astronomy Department, West Virginia University, Morgantown, WV 26506-6315, USA \\ Correspondence: Leonardo Golubović, Physics and Astronomy Department, West Virginia University, POB 6315, \\ Morgantown, WV 26506, USA. E-mail: lgolubov@wvu.edu
}

Received: July 18, 2017

Accepted: July 28, 2017

Online Published: August 21, 2017

doi:10.5539/apr.v9n5p12

URL: https://doi.org/10.5539/apr.v9n5p12

\begin{abstract}
The physics of manmade celestial scale objects, such as Space Elevators connecting the Earth with outer space, has recently attracted increased attention of diverse researchers. In this article we review basic physics of celestial scale dumbbells such as the Analemma Tower suspended from an asteroid orbiting the Earth (Clouds, 2017). Celestial dumbbells involve two large masses (top and bottom) connected by strings. The two masses move geosynchronously with the Earth, with the bottom mass remaining close to the Earth and the top mass moving above the Earth's geosynchronous satellite orbit. Appealing examples of celestial scale dumbbells are untied Rotating Space Elevators (RSE) (Knudsen \& Golubović, 2015). Physics of untied rotating space elevators. European Physical Journal Plus 130, 243.]. Celestial scale dumbbells exhibit rich and interesting nonlinear dynamics caused by instabilities of dumbbell geosynchronous motion discussed in this review article. We also point out that celestial scale dumbbells are physically feasible (in terms of nowadays available materials strengths) on dwarf planets in the main asteroid belt of the Solar system such as Ceres.
\end{abstract}

Keywords: Space Elevator, Classical Mechanics, Space Travel, Nonlinear Dynamics, Instabilities and Transitions

\section{Introduction}

Visions of manmade celestial scale objects, such as Space Elevators connecting the Earth with outer space, have attracted increased attention of diverse researchers in this millennium (Edwards \& Westling, 2003). Discoveries of ultra-strong materials such as carbon nanotubes (Yu et al., 2000a, Yu et al., 2000b) and diamond nano-thread structures (Fitzgibbons et al., 2014) have provided new stimulus in this field of research. Space elevators are celestial scale examples of physical systems with reduced dimensionality such as the strings, polymers, and membranes (Kardar, 2007; Nelson, 2002; Nelson, Piran, \& Weinberg, 1988). The classical and statistical mechanics of space elevators represents a bold extension of previous studies of artificial satellite dynamics such as those of Beletskii (1965) and Hughes (2012).

Recently, a new venue has emerged in space elevator physics: Rotating Space Elevator (RSE) (Golubovic \& Knudsen, 2009; Knudsen \& Golubovic, 2014; Knudsen \& Golubovic, 2015; Knudsen \& Golubovic, 2016; Golubovic \& Knudsen, 2017). The RSE is a double rotating floppy string reaching extraterrestrial locations. Figure 1(a) depicts elliptical version of RSE. In this figure, the coordinate system $\left(R_{1}, R_{2}, R_{3}\right)$ rotates together with the Earth around the $R_{2}$-axis (not shown) pointing through the north pole N. The RSE executes an internal rotation (nearly around the $R_{1}$-axis) with period $T_{\text {RSE }}$ as well as geosynchronous (together with the Earth) rotation with the period one sidereal day. Interestingly, objects sliding along the RSE string (climbers) do not require internal engines or propulsion to be transported far away from the Earth's surface. The RSE thus solves a major problem in space elevator physics which is how to supply energy to the climbers moving along space elevator strings.

In this review article we discuss basic physics of celestial scale dumbbells such as the Analemma Tower suspended from an asteroid orbiting the Earth (Clouds, 2017). Celestial dumbbells involve two large masses (top and bottom) connected by strings. The two masses move geosynchronously with the Earth, with the bottom mass remaining close to the Earth and the top mass moving above the Earth's geosynchronous satellite orbit. Notable and unusual examples of celestial scale dumbbells are untied Rotating Space Elevators (RSE) (Knudsen \& Golubović, 2015). RSE is celestial scale double rotating floppy loop, with a large mass concentration in the top and bottom regions of the loop; see figure 1(b). The figure depicts RSE mass line density $\mu(s)$ versus the arc-length distance $s$ from the RSE bottom (obtained by Eq. (8) of Golubovic \& Knudsen (2009)) of the elliptic RSE with the shape as 
in Figure 1(a) for the RSE period of $T_{R S E}=7.04 \mathrm{~min}$, minor elliptical semi-axis $b=0.17$ Earth radii, and the gap $D=0.1564$ Earth radii. Note that the RSE mass is largely concentrated in the top and bottom regions of the RSE.

a)

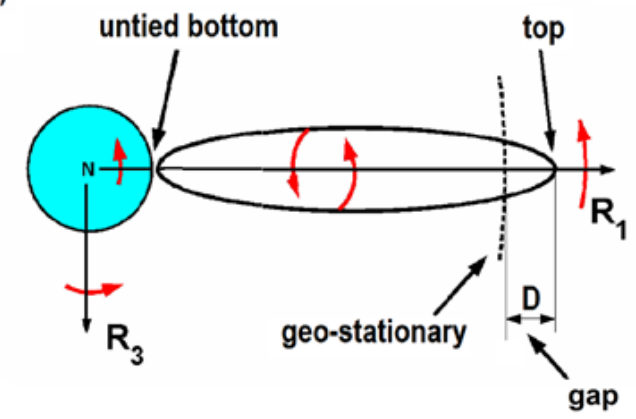

b)
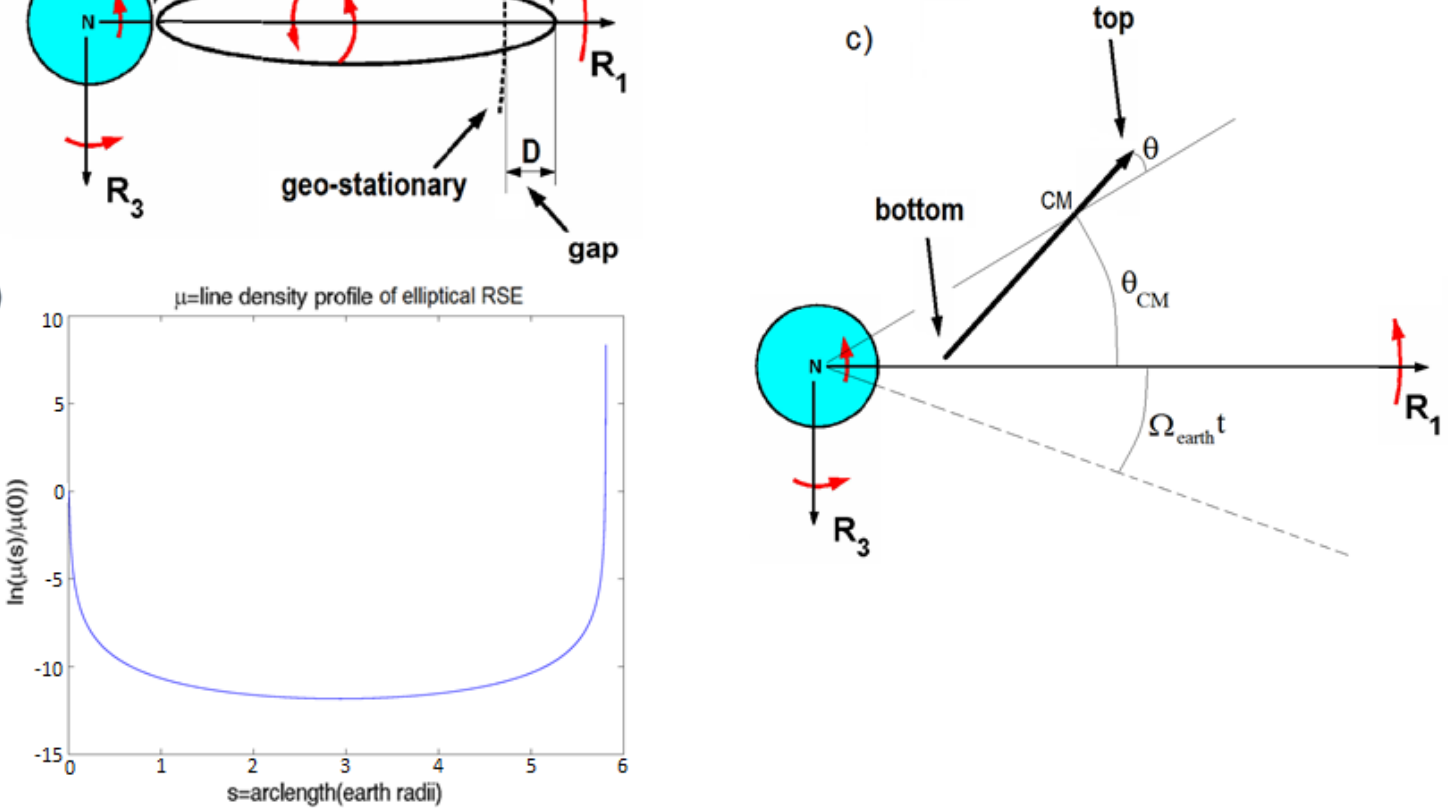

Figure 1. Adapted from Knudsen \& Golubovic (2015): (a) Elliptical version of RSE. The RSE bottom is close to the Earth surface but it is not tied to it. The RSE top is at the distance $D$ ("gap") above the geostationary satellite orbit (with the radius of 6.6108 Earth radii). In (b), RSE magical mass line density $\mu(s)$ versus the arc-length

distance $s$ from the RSE bottom. (c) Untied RSE projection onto the equatorial plane $\left(R_{1}, R_{3}\right)$ of the geosynchronous frame which rotates with the angular velocity $\Omega_{\text {earth }}$ with respect to the inertial frame (dashed axis). The RSE is conceptualized as an arrow with the arrow's head being the RSE top and the arrow's end of tail being the RSE bottom

Celestial dumbbells exhibit interesting nonlinear dynamics. Thus, the untied RSE loop may still behave as if it were tied to the planet. Such a quasi-tied yet untied RSE loop remains close to the Earth and exhibits persistent shape and enduring double rotating motion. Moreover, the climbers sliding along such a quasi-tied RSE move in much the same way as they do along a tied RSE.

Under some conditions however geosynchronous orbits of untied celestial scale dumbbells, such as untied RSE and Analemma Tower, may undergo an instability leading them to a dynamical state in which the dumbbell hops well above the Earth surface; see figure 2. This hopping regime occurs if the dumbbell top initial height above geostationary orbit (called as "gap" $D$ in Figure 1 ) is bigger than $D_{\text {hopping }} \approx 0.2$ Earth radii. By increasing the gap above the hopping threshold, the maximum height reached during hopping increases and eventually diverges at the characteristic gap value $D_{\text {unbinding }} \approx 2.1$ Earth radii. Such a celestial scale dumbbell unbinds from the Earth and escapes to infinity, i.e., to interplanetary space. This rich and interesting dynamics of celestial scale dumbbells is discussed in the present review article. 

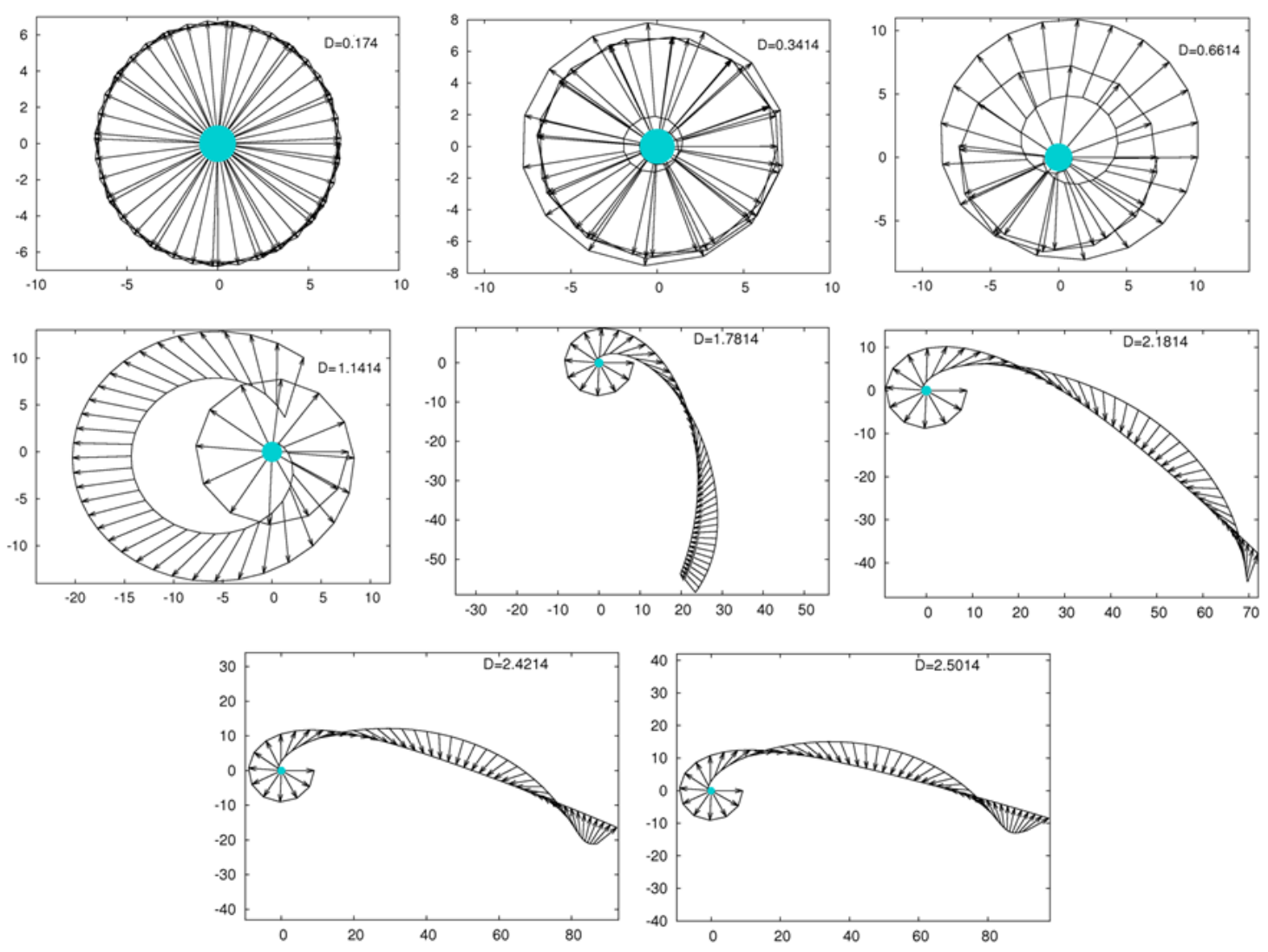

Figure 2. From simulations of floppy RSE, Knudsen \& Golubovic (2015). Each panel gives a time sequence of RSE bottom and top projections onto the equatorial plane in the inertial frame, over the first $6000 \mathrm{~min}$ of time evolution. The length unit used here is 1 Earth radius. In all panels $b=0.17$ Earth radii, $T_{R S E}=7.04 \mathrm{~min}$. The Earth is depicted as a small circle. The RSE is conceptualized as an arrow with head being the RSE top and end of tail being the RSE bottom

In figure 2, displayed is one panel with $D<D_{\text {hopping }} \cong 0.2$ Earth radii, when the untied RSE is quasi-tied and nearly follows the Earth rotation. In the figure, there are four panels for the gaps $\mathrm{D}$ in the range between $D_{\text {hopping }} \cong 0.2$ Earth radii and $D_{\text {unbinding }} \cong 2.11$ Earth radii when the untied RSE exhibits a hopping motion (in two of the panels, the hopping period is longer than the displayed 6000min evolution). The remaining three panels are for the gaps $D$ in the range above $D>D_{\text {unbinding }} \cong 2.11$ Earth radii when the untied RSE escapes from the Earth to infinity.

We note that various analytic results displayed in this review are given in the form applicable to celestial scale dumbbells deployed around any rotating planet. The Earth was used just as an example. However, practically significant examples are actually dwarf planets of the Solar system such as Ceres, Vesta, Pallas, and Juno. As discussed in section 6, celestial scale dumbbells are physically feasible (in terms of nowadays available materials tensile strengths) on the dwarf planets in the main asteroid belt of the Solar system.

\section{The Physics of Celestial Scale Dumbbells}

By figure 1(b), RSE string has large mass concentration in the top and bottom regions of the RSE loop. This feature motivates to model the floppy RSE string as a dumbbell comprised of two point masses, $M$, the mass of the RSE top and $m$, the mass of the RSE bottom. Within this model, the length of the dumbbell $l$, corresponding to the top to bottom distance, is assumed to be constant. In the floppy RSE simulations (Knudsen \& Golubovic, 2015), the top-to-bottom distance indeed exhibits only very small oscillations in the quasi-tied regime, and relatively small changes in hopping/unbinding regimes if the RSE is not too far from the Earth, see Figure 2. Thus, interestingly, a simple dumbbell model provides some quantitatively very accurate results not only for the RSE quasi-tied regime but also for the RSE hopping and unbinding regime; see Knudsen \& Golubovic (2015). 
On historic side, we note that the dynamics of rigid objects such as artificial satellites has been subject of extensive studies; see Beletskii (1965) and Hughes (2012) for review. Previous studies were primarily concerned with small size satellites. Notable exception is a heuristic discussion made by Duboshin (see Beletskii, 1965) which will be addressed later on in this review; see section 5. The dumbbells that we discuss here are of celestial scale, as in figures 1 and 2, with the bottom close to the Earth and far away from the dumbbell top which is above the geostationary orbit at $r_{\text {geo }}=6.6108 R_{E} \gg R_{E}$, with $R_{E}=$ Earth radius. This separation of length scales requires a special care and detailed analytic results discussed in this review.

a)

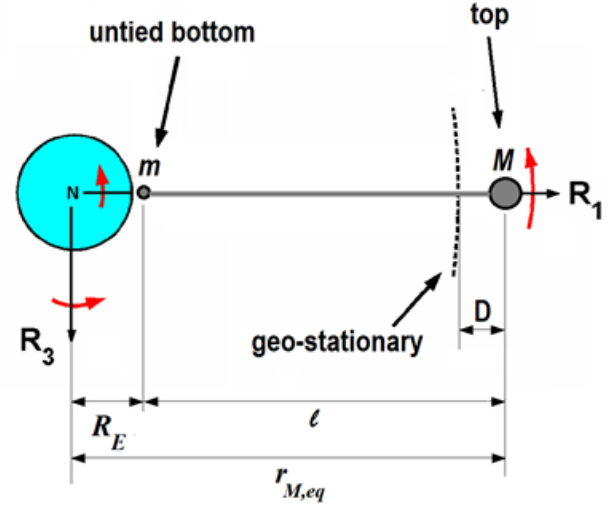

b)

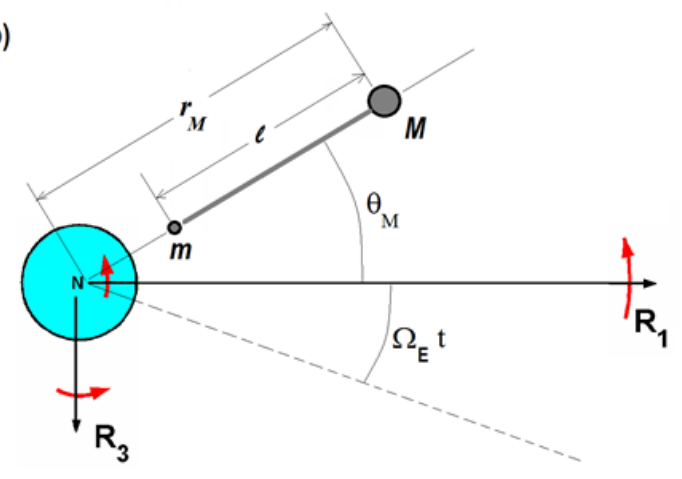

Figure 3. (a) Dumbbell in the dynamical equilibrium state in geosynchronous frame. The coordinate system $\left(R_{1}, R_{2}, R_{3}\right)$ rotates together with the Earth around the $R_{2}$-axis (not shown) pointing through the north pole $\mathrm{N}$. In (b), the degrees of freedom $r_{M}$ and $\theta_{M}$ used in the Lagrangian in Eq. (A9) are indicated. The dashed axis is static in the inertial frame. Adapted from Knudsen \& Golubovic (2015)

The RSE in Figure 1(a) corresponds to the dumbbell dynamical equilibrium state realized in the geosynchronous frame, Figure 3(a). In this state, the total of centrifugal and gravitational forces (of the Earth) on the dumbbell masses $m$ (bottom mass) and $M$ (top mass) vanishes,

$$
0=m\left(\Omega_{E}^{2} R_{E}-\frac{G M_{E}}{R_{E}^{2}}\right)+M\left(\Omega_{E}^{2} r_{M, e q}-\frac{G M_{E}}{r_{M, e q}^{2}}\right) .
$$

Here, $G$ is Newton's gravitational constant while $M_{E}, R_{E}, \Omega_{E}=2 \pi / T_{d a y}$ ( $T_{d a y}=$ sidereal day), are respectively the mass, radius and the angular velocity of the Earth (seen in the inertial frame), while $r_{M, e q}$ is the equilibrium radial distance of the dumbbell top with the mass $M$. The length of the dumbbell is thus

$$
l=r_{M, e q}-R_{E}
$$

Equation (1) holds even if the dumbbell bottom is set above the surface of the Earth, in which case $R_{E}$ signifies the equilibrium radial distance of the bottom mass $m$. It will be convenient to express our results in terms of the radius of the geo-synchronous satellite orbit, $r_{\text {geo }}$, satisfying the relation

$$
G M_{E}=\Omega_{E}^{2} r_{g e o}^{3} .
$$

By Equations (3) and (1),

$$
0=m\left(R_{E}-\frac{r_{g e o}^{3}}{R_{E}^{2}}\right)+M\left(r_{M, e q}-\frac{r_{g e o}^{3}}{r_{M, e q}^{2}}\right) .
$$


By Equation (4), the dynamical equilibrium condition requires a specially chosen value of the bottom to top mass ratio,

$$
\frac{m}{M}=\frac{r_{M, e q} R_{E}^{2}}{r_{\text {geo }}^{3}} \frac{1-\left(\frac{r_{\text {geo }}}{r_{M, e q}}\right)^{3}}{1-\left(\frac{R_{E}}{r_{\text {geo }}}\right)^{3}} .
$$

By Equation (5), positivity of the $m / M$ ratio implies that $R_{E}<r_{\text {geo }}<r_{M, e q}$, i.e., the bottom (top) mass must be below (above) the geo-synchronous satellite orbit. In our case, $r_{g e o}=6.6108$ Earth radii, so in the denominator of Equation (5) one has the small quantity

$$
\left(\frac{R_{E}}{r_{\text {geo }}}\right)^{3}=6.6108^{-3}=3.46 \times 10^{-3}
$$

which is, in terms of Equation (1), the ratio between the inertial (centrifugal) and gravitational force on the bottom mass m. Ignoring this small quantity reduces Equation (5) to,

$$
\frac{m}{M} \cong \frac{r_{M, e q} R_{E}^{2}}{r_{\text {geo }}^{3}}\left[1-\left(\frac{r_{g e o}}{r_{M, e q}}\right)^{3}\right]=\left(\frac{R_{E}}{r_{\text {geo }}}\right)^{2}\left(1+\frac{D}{r_{\text {geo }}}\right)\left[1-\left(1+\frac{D}{r_{\text {geo }}}\right)^{-3}\right] .
$$

where we introduced the gap distance $D=r_{M, e q}-r_{\text {geo }}$; see figures 1 (a) and 3(a). For $D<<r_{\text {geo }}$, by expanding terms in Equation (7),

$$
\frac{m}{M} \cong 3\left(\frac{R_{E}}{r_{\text {geo }}}\right)^{2} \frac{D}{r_{\text {geo }}}=3\left(\frac{R_{E}}{r_{\text {geo }}}\right)^{3} \frac{D}{R_{E}}
$$

By recalling here that, for example, $D_{\text {hopping }} / R_{E} \cong 0.2$, in view of Equation (6), the bottom to top mass ratio in Equation (8) is typically very small quantity for physically interesting situations.

To discuss dumbbell dynamics out of the equilibrium state in figure 3(a), one needs to invoke the full set of dumbbell equations of motion. They can be generated in a standard way, by employing classical Lagrangian $L=K E-P E$, with dumbbell kinetic and gravitational potential energy expressed in terms of suitable coordinates, such as the angles $\theta(t)$ and $\theta_{c m}(t)$ (defined in Figure 1(c)), dumbbell center of mass (cm) radial distance, and the out of plane coordinates $\varphi(t)$ and $\left(R_{2}\right) \mathrm{cm}$ (dumbbell tilt tangle and $\mathrm{cm}$ displacement away from equatorial plane). We will review the results of this general and rather complex approach in section 4 . In this section and section 3, we discuss a simple approach of Knudsen and Golubovic (2015), which captures all major aspects of celestial dumbbell dynamics such as the hopping and unbinding regimes of motion. The approach is based on the same approximation as done in the transition from Equation (5) to Equation (7), which is to ignore inertial relative to gravitational effects of the bottom mass $m$. See Equation (6) and the above discussions. This is tantamount to ignoring the kinetic energy of the bottom mass while maintaining its gravitational potential energy in the dumbbell Lagrangian. In addition, one sets the angle $\theta(t)$ defined in Figure 1(c) to be zero as if the bottom is suspended from the top slowly moving in geosynchronous frame, see Figure 3(b). This constraint is motivated by RSE simulations of Knudsen and Golubovic (2015) which indeed show that $\theta(t)$ is very small in quasi-tied states; see Figure 2. Moreover, this angle remains small even in the hopping state, at least while the dumbbell is relatively close to the Earth; see Figure 2. With the above two assumptions, the resulting Lagrangian $L=K E-P E$ (dumbbell kinetic minus gravitational potential energy in the Earth inertial frame) has the simple form,

$$
L\left(r_{M}, \dot{r}_{M}, \dot{\theta}_{M}\right)=\frac{M}{2}\left[\left(\dot{r}_{M}\right)^{2}+r_{M}^{2}\left(\Omega_{E}+\dot{\theta}_{M}\right)^{2}\right]+\frac{G M_{E} M}{r_{M}}+\frac{G M_{E} m}{r_{M}-l} .
$$

Here, as in Figure 3(b), $r_{M}$ is the radial distance of the top mass $M$ and $\theta_{M}$ is the azimuthal angle of the top in the geo-synchronous frame; note that top angular velocity in inertial frame is $\Omega_{E}+\dot{\theta}_{M}$. The first two terms in the 
Equation (9) are just the standard Lagrangian for the motion of the object of mass $M$ (the top) in the Earth gravitational field. The last term in Equation (9) comes from the gravitational potential energy of the bottom mass $m$ which is at the radial distance $=r_{M}-l$ from the Earth center; see Figure 3(b). By Equation (9), the main effect of the bottom presence is to modify the form of the central potential seen by the dumbbell top. Resulting Lagrangian dynamics of the dumbbell top thus has standard form of motion in a central potential, with a conserved angular momentum,

$$
\frac{\partial L}{\partial \dot{\theta}_{M}}=M r_{M}^{2}(t)\left[\Omega_{E}+\dot{\theta}_{M}(t)\right]=M r_{M}^{2}(0)\left[\Omega_{E}+\dot{\theta}_{M}(0)\right],
$$

and a conserved mechanical energy function,

$$
\begin{aligned}
& E=K E+P E=\frac{M}{2}\left[\left(\dot{r}_{M}\right)^{2}+r_{M}^{2}\left(\Omega_{E}+\dot{\theta}_{M}\right)^{2}\right]-\frac{G M_{E} M}{r_{M}}-\frac{G M_{E} m}{r_{M}-l} \\
& =\frac{M}{2}\left(\dot{r}_{M}\right)^{2}+U_{e f f}\left(r_{M}\right)=\frac{M}{2}\left(\dot{r}_{M}(0)\right)^{2}+U_{e f f}\left(r_{M}(0)\right)
\end{aligned}
$$

Here, we introduced the effective central potential,

$$
U_{e f f}\left(r_{M}\right)=-\frac{G M_{E} M}{r_{M}}-\frac{G M_{E} m}{r_{M}-l}+\frac{M r_{M}^{4}(0)\left[\Omega_{E}+\dot{\theta}_{M}(0)\right]^{2}}{2 r_{M}^{2}},
$$

or, by Equation (3),

$$
U_{e f f}\left(r_{M}\right)=M \Omega_{E}^{2}\left[-\frac{r_{g e o}^{3}}{r_{M}}-\frac{r_{g e o}^{3}}{r_{M}-l} \frac{m}{M}+\frac{r_{M}^{4}(0)\left[1+\dot{\theta}_{M}(0) / \Omega_{E}\right]^{2}}{2 r_{M}^{2}}\right] .
$$

By Equations (9) and (10), the Euler-Lagrange equation of motion for $r_{M}$ has the form,

$$
\begin{aligned}
& M \ddot{r}_{M}(t)=-\frac{G M_{E} M}{r_{M}^{2}(t)}-\frac{G M_{E} m}{\left(r_{M}(t)-l\right)^{2}}+M r_{M}(t)\left[\Omega_{E}+\dot{\theta}_{M}(t)\right]^{2} \\
& =-\left.\frac{\partial U_{e f f}}{\partial r_{M}}\right|_{r_{M}=r_{M}(t)}=-\frac{G M_{E} M}{r_{M}^{2}(t)}-\frac{G M_{E} m}{\left(r_{M}(t)-l\right)^{2}}+\frac{M r_{M}^{4}(0)\left[\Omega_{E}+\dot{\theta}_{M}(0)\right]^{2}}{r_{M}^{3}(t)}
\end{aligned}
$$

The simplest solutions of Equation (14) are dynamic equilibrium states which are circular orbits with constant $r_{M}(t)=r_{M}(0)$ and $\dot{\theta}_{M}(t)=\dot{\theta}_{M}(0)$. For them, by Equation (14)

$$
-\left.\frac{\partial U_{e f f}}{\partial r_{M}}\right|_{r_{M}=r_{M}(0)}=-\frac{G M_{E} M}{r_{M}^{2}(0)}-\frac{G M_{E} m}{\left(r_{M}(0)-l\right)^{2}}+M r_{M}(0)\left[\Omega_{E}(0)+\dot{\theta}_{M}(0)\right]^{2}=0,
$$

or, by Equation (3),

$$
-\frac{r_{g e o}^{3}}{r_{M}^{2}(0)}-\frac{r_{\text {geo }}^{3}}{\left(r_{M}(0)-l\right)^{2}} \frac{m}{M}+r_{M}(0)\left[1+\dot{\theta}_{M}(0) / \Omega_{E}\right]^{2}=0
$$

A dumbbell in this dynamic equilibrium state is at rest in the geosynchronous frame if $\dot{\theta}_{M}(0)=0$. For this case, Equation (16) yields the relation,

$$
\frac{m}{M}=\frac{\left(r_{M}(0)-l\right)^{2} r_{M}(0)}{r_{g e o}^{3}}\left[1-\left(\frac{r_{g e o}}{r_{M}(0)}\right)^{3}\right]
$$

which is identical to Equation (7), by recalling Equation (2), i.e.,

$$
l=r_{M}(0)-R_{E}=r_{\text {geo }}+D-R_{E} .
$$

By Equation (14), small harmonic oscillations, $\delta r_{M}(t)=r_{M}(t)-r_{M}(0)$ around this dynamic equilibrium state obey the linearized equation of motion 


$$
M \frac{d^{2} \delta r_{M}(t)}{d t^{2}}=-\left.\frac{\partial^{2} U_{e f f}}{\partial r_{M}{ }^{2}}\right|_{r_{M}=r_{M}(0)} \delta r_{M}(t) .
$$

By Equation (19), angular frequency of this small oscillation (slow dumbbell mode) obeys the relation

$$
\Omega_{\text {slow }}^{2}=\left.M^{-1} \frac{\partial^{2} U_{e f f}}{\partial r_{M}^{2}}\right|_{r_{M}=r_{M}(0)},
$$

which is by Equation (13) [with $\dot{\theta}_{M}(0)=0$ ], and Equations (17) and (18), found to be

$$
\begin{aligned}
& \left(\frac{\Omega_{\text {slow }}}{\Omega_{E}}\right)^{2}=1-2 \frac{r_{M}(0)-R_{E}}{R_{E}}\left[1-\left(\frac{r_{g e o}}{r_{M}(0)}\right)^{3}\right] \\
& =1-2 \frac{r_{\text {geo }}+D-R_{E}}{R_{E}}\left[1-\left(\frac{r_{g e o}}{r_{\text {geo }}+D}\right)^{3}\right]
\end{aligned}
$$

The slow mode frequency Equation (21) as function of the gap $D$ is displayed in Figure 4(b). This theoretical result for rigid dumbbell agrees very well with the corresponding result obtained from the simulations of the floppy untied RSE of Knudsen and Golubovic (2015). By Equation (21), $\Omega_{\text {slow }} \rightarrow \Omega_{E}$ for $D \rightarrow 0$. Importantly, by Equation (21) with $r_{\text {geo }}=6.6108 R_{E}$, one finds that $\Omega_{\text {slow }} \rightarrow 0$ for $D \rightarrow D_{\text {hopping }}=0.20123 R_{E}$ in accord with the observed complete softening of the slow mode from the RSE simulations seen in Figure 5 of Knudsen and Golubovic (2015). For $D>D_{\text {hopping }}$, the slow mode becomes unstable and hopping regime of motion sets in; see section 3. By Figure 4(b), $\Omega_{\text {slow }}^{2}$ appears as a nearly linear function of the gap $D$. In fact, the result in Equation (21) can be expanded in powers of the small parameter $R_{E} / r_{\text {geo }}$, with the result

$$
\left(\frac{\Omega_{\text {slow }}}{\Omega_{E}}\right)^{2}=1-6 \bar{D}+\frac{R_{E}}{r_{\text {geo }}}\left(6 \bar{D}+6 \bar{D}^{2}\right)+\left(\frac{R_{E}}{r_{\text {geo }}}\right)^{2}\left(-12 \bar{D}^{2}-8 \bar{D}^{3}\right)+O\left(\frac{R_{E}}{r_{\text {geo }}} \bar{D}\right)^{3},
$$

with

$$
\bar{D}=\frac{D}{R_{E}}
$$

Eq. (22) can be iteratively solved to find the $\bar{D}_{\text {hopping }}=D_{\text {hopping }} / R_{E}$ for which $\Omega_{\text {slow }} \rightarrow 0$, with the result having the form of an expansion in powers of the small parameter $R_{E} / r_{\text {geo }}$,

$$
\bar{D}_{\text {hopping }}=\frac{D_{\text {hopping }}}{R_{E}}=\frac{1}{6}+\frac{7}{36} \frac{R_{E}}{r_{\text {geo }}}+\left(\frac{2}{3}\right)^{4}\left(\frac{R_{E}}{r_{\text {geo }}}\right)^{2}+O\left(\frac{R_{E}}{r_{\text {geo }}}\right)^{3} .
$$

After truncating out the $\left(R_{E} / r_{\text {geo }}\right)^{3}$ term, Equation (24) yields $\bar{D}_{\text {hopping }}=D_{\text {hopping }} / R_{E}=0.20060$ for $r_{\text {geo }}=6.6108 R_{E}$, versus the aforementioned result $\bar{D}_{\text {hopping }}=D_{\text {hopping }} / R_{E}=0.20123$ obtained by numerically solving Equation (21) with $\Omega_{\text {slow }}=0$. It is interesting to note that, by Equation (24), in the limit of slowly rotating planets $R_{E} / r_{\text {geo }} \rightarrow 0$, the $\bar{D}_{\text {hopping }}=D_{\text {hopping }} / R_{E}$ has a finite universal value $=1 / 6$. For $\bar{D}<\bar{D}_{\text {hopping }}<1$, from the specific form of the expansion Equation (22) [with higher order terms in $\bar{D}$ going with even higher order terms in $R_{E} / r_{\text {geo }}$ ] it is evident that the terms nonlinear in $\bar{D}$ give only a minor contribution for a small $R_{E} / r_{g e o}$. Thus, to a good approximation for $\bar{D}<\bar{D}_{\text {hopping }}$,

$$
\left(\frac{\Omega_{\text {slow }}}{\Omega_{E}}\right)^{2} \approx 1-6\left(1-\frac{R_{E}}{r_{\text {geo }}}\right) \bar{D}=1-\frac{\bar{D}}{\bar{D}_{\text {hopping }}},
$$

i.e., the $\Omega_{\text {slow }}^{2}$ is nearly a linear function of the gap $D$, as evidenced from its plot in Figure 4(b). By Equation (25), $\bar{D}_{\text {hopping }} \approx 1 /\left(6-6 R_{E} / r_{\text {geo }}\right)$, which for $r_{\text {geo }}=6.6108 R_{E}$ yields a sound approximation $\bar{D}_{\text {hopping }} \approx 0.19637$. In section 4 , we will revisit the result for $\bar{D}_{\text {hopping }}^{\text {geo }}$ within the exact dumbbell model going beyond the approximations 
involved in the theory based on the approximate Lagrangian Equation (9). It turns out that the results in Equations (22) and (24) are actually exact to the second order in $R_{E} / r_{\text {geo }}$, as displayed in these two equations.

\section{Hopping and Unbinding of Celestial Scale Dumbbells}

Let us discuss the hopping and unbinding regimes of dumbbell motion seen in the simulations of RSE in figure 2 . We will elucidate these striking phenomena within the approximate dumbbell Lagrangian in Equation (9). By mechanical energy conservation Equation (11), in combination with the Equation (13) [with $\dot{\theta}_{M}(0)=0$ ], and Equation (17), one finds,

$$
\frac{1}{2}\left(\dot{r}_{M}\right)^{2}-\frac{1}{2}\left(\dot{r}_{M}(0)\right)^{2}=-\frac{1}{M}\left[U_{e f f}\left(r_{M}\right)-U_{e f f}\left(r_{M}(0)\right)\right]
$$

with,

$$
\begin{aligned}
& U_{e f f}\left(r_{M}\right)-U_{e f f}\left(r_{M}(0)\right) \\
& =M \Omega_{E}^{2} \frac{r_{M}^{2}(0) l}{2} \cdot \frac{\left[r_{M}-r_{M}(0)\right]^{2}}{r_{M}\left(r_{M}-l\right)} \cdot\left(\frac{Z}{r_{M}(0)}-\frac{1}{r_{M}}\right)
\end{aligned}
$$

where

$$
Z=\frac{r_{M}(0)}{l}-2\left[1-\left(\frac{r_{g e o}}{r_{M}(0)}\right)^{3}\right]
$$

or, by Equation (18),

$$
Z=Z(D)=\frac{r_{\text {geo }}+D}{r_{\text {geo }}+D-R_{E}}-2\left[1-\left(\frac{r_{\text {geo }}}{r_{\text {geo }}+D}\right)^{3}\right] .
$$

We plot the function $Z(D)$ in Figure 4(a), for the interesting case with $r_{g e o}=6.6108 R_{E}$. Implications of the results in Equations (26-29) are best understood from the plots of the effective potential Equation (27) displayed in the panels (c) and (d) of Figure 4.

a)

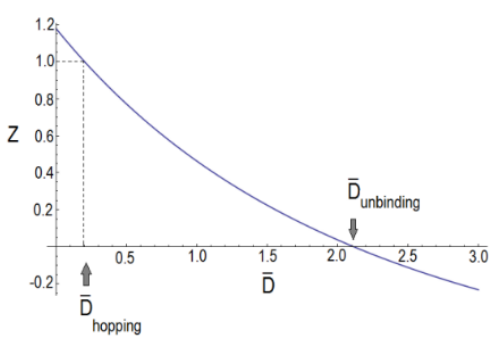

c)

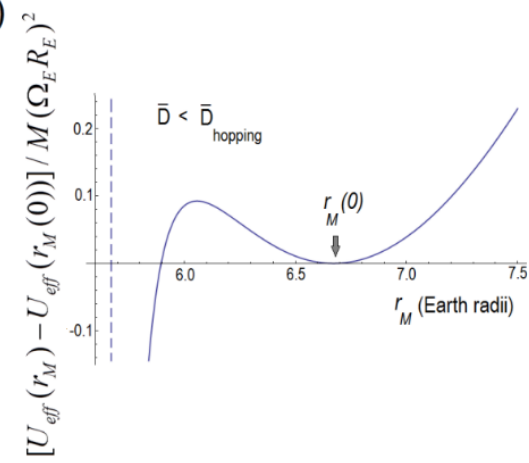

b)

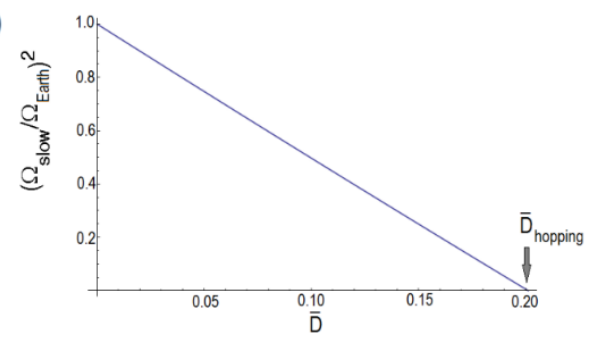

d)

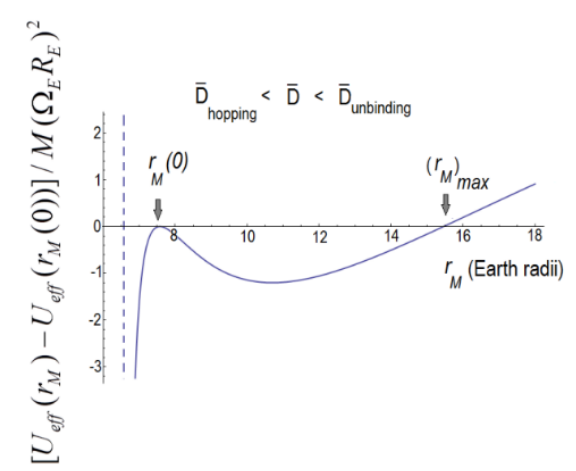

Figure 4. Adapted from Knudsen \& Golubovic (2015): (a) Function $\mathrm{Z}$ versus $\bar{D}=D / R_{E}$; see Equation (29). (b) Slow mode angular frequency (squared) versus $\bar{D}=D / R_{E}$; see Equations (21) and (30). (c) Case $\bar{D}<\bar{D}_{\text {hopping }}$ : form of the effective potential Equation (27) for $\bar{D}=0.05$. (d) Case $\bar{D}_{\text {hopping }}<\bar{D}<\bar{D}_{\text {unbinding }}$ : form of the effective potential Equation (27) for $\bar{D}=0.95$. In all panels, $r_{\text {geo }}=6.6108 R_{E}$ 
Thus, in Figure 4(c) we see that for $D<D_{\text {hopping }}$ [whence $Z(D)>1$ ] the potential $U_{\text {eff }}\left(r_{M}\right)$ has stable minimum at $r_{M}=r_{M}(0)$. This situation corresponds to the quasi-tied state with a stable slow mode with the frequency given by Equation (21). This equation can be easily shown [by Equation (29)] to be equivalent to

$$
\left(\frac{\Omega_{\text {slow }}}{\Omega_{E}}\right)^{2}=\frac{r_{\text {geo }}+D-R_{E}}{R_{E}} \cdot(Z(D)-1) .
$$

For $D \rightarrow D_{\text {hopping }}$, the $Z(D) \rightarrow 1$, so the slow mode completely softens in this limit; see figs. 4(a) and (b). By Figure 4(d), for $D>D_{\text {hopping }}$ [whence $Z(D)<1$ ] the effective potential $U_{\text {eff }}\left(r_{M}\right)$ has unstable maximum at $r_{M}=r_{M}(0)$ (a homoclinic point) corresponding, by Equation (30), to an unstable slow mode. This situation corresponds to the hopping RSE state seen in the simulations of Knudsen \& Golubovic (2015) in Figure 2, with $r_{M}(t)$ rising above $r_{M}(0)$ all the way up to a turning point where momentarily $\dot{r}_{M}(t)=0$. In the case with $\dot{r}_{M}(0) \approx 0$, by Equations (26) and (27) [see also Figure 4(d)], at the turning point, the $r_{M}(t)$ reaches its maximum value given by

$$
\left(r_{M}\right)_{\max }=\frac{r_{M}(0)}{Z(D)}=\frac{r_{g e o}+D}{Z(D)} .
$$

Thus,

$$
Z(D)=\frac{r_{M}(0)}{\left(r_{M}\right)_{\max }}
$$

Importantly, $Z(D)$ crosses zero at a characteristic value of $D$; see figure 4(a). This corresponds to the unbinding transition point at which $\left(r_{M}\right)_{\max } \rightarrow \infty$ and the dumbbell approaches infinity. This transition happens at the characteristic $D$ value solving the equation $Z\left(D_{\text {unbinding }}\right)=0$. For the interesting case with $r_{\text {geo }}=6.6108 R_{E}$, one finds, by Equation (A29), that $Z(D) \rightarrow 0$, i.e., $\left(r_{M}\right)_{\max } \rightarrow \infty$ for $D \rightarrow D_{\text {unbinding }}=2.1123 R_{E}$. Remarkably, this result obtained from rigid dumbbell model is in a very good agreement with the simulations of the floppy untied RSE which indicate that $D_{\text {unbinding }} \approx 2.11 R_{E}$; see Figure 9 of Knudsen \& Golubovic (2015). In this figure, they employed the center of mass radial distance $r_{c m}$ rather than the top radial distance $r_{M}$, yet the difference between the two is very small due to the small $m / M$ ratio; see Equation (8). From Figure 9 of Knudsen \& Golubovic (2015), one can see that the ratio $r_{c m}(0) /\left(r_{c m}\right)_{\max }$ from the simulations of floppy untied RSE is strikingly well approximated by the function $Z(D)$ in Equation (29) (plotted in Figure 4(a)) which was obtained from the rigid dumbbell model.

At the unbinding threshold, $D=D_{\text {unbinding }}$ [whence $Z=0$ ], Equations (26) [with $\dot{r}_{M}(0)=0$ ] and (29) can be easily used to show that $\dot{r}_{M}(t) \sim t^{-1 / 3}$, i.e., $r_{M}(t) \sim t^{2 / 3}$ at long times, in accord with RSE simulations results displayed in Figure 10 of Knudsen \& Golubovic (2015). Above the unbinding threshold, $D>D_{\text {unbinding }}$ [whence $\left.Z(D)<0\right]$, Equations (27) [with $\dot{r}_{M}(0)=0$ ] and (29) can be used to show that, at long times, $\dot{r}_{M}(t=\infty) \sim[-Z(D)]^{1 / 2} \sim \sqrt{D-D_{\text {unbinding }}}$ (for $D$ slightly above $D_{\text {unbinding }}$ ). So the dumbbell reaches infinity with a finite escape velocity, in accord with the RSE simulations results of Knudsen \& Golubovic (2015) displayed in their figure 10 .

The equation $Z\left(D_{\text {unbinding }}\right)=0$ can be solved for $D_{\text {unbinding }}$ by an expansion in powers of the small parameter $R_{E} / r_{\text {geo }}$. One thus finds, to the second order in $R_{E} / r_{\text {geo }}$,

$$
\frac{D_{\text {unbinding }}}{r_{\text {geo }}}=2^{1 / 3}-1+\frac{1}{3} \frac{R_{E}}{r_{\text {geo }}}+\frac{2^{5 / 3}}{9}\left(\frac{R_{E}}{r_{\text {geo }}}\right)^{2}+O\left(\frac{R_{E}}{r_{\text {geo }}}\right)^{3} .
$$

To this order, for $r_{\text {geo }}=6.6108 R_{E}$, the truncated Equation (33) gives $D_{\text {unbinding }} \approx 2.1050 R_{E}$ in a reasonable agreement with the aforementioned numerical solution $D_{\text {unbinding }}=2.1123 R_{E}$. We will return to the discussions of the dumbbell unbinding transition in section 5. 


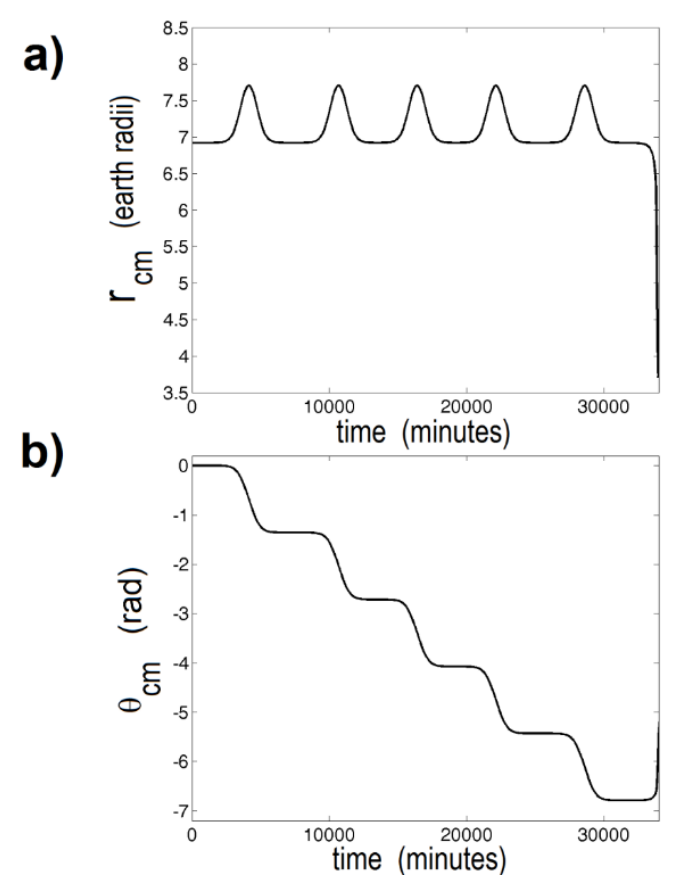

Figure 5. From simulations of floppy RSE, Knudsen \& Golubovic (2015). The dynamics of the RSE center of mass in terms of its center of mass coordinates $r_{c m}(t)$ in (a) and $\theta_{c m}(t)$ in (b), for the hopping RSE with gap $D=0.3164$ Earth radii, $\stackrel{c}{b}=0.17$ Earth radii, $T_{R S E}=7.04 \mathrm{~min}$

Let us discuss in more detail the dumbbell hopping regime of motion. The existence of hopping dumbbell motion reflects genuine instability of dumbbell geosynchronous orbits occurring for $D>D_{\text {hopping }}$ in contrast to stable circular orbits occurring for $D<D_{\text {hopping }} \approx 0.2$ Earth radii where untied dumbbells are quasi-tied and geosynchronously follow the Earth rotation with dumbbell bottom remaining close to the Earth; see Figure 2 . However, for $D>D_{\text {hopping }}$, in Figure 2 one can see that the untied RSE bottom hops away from the Earth surface.

Details of this hopping are displayed in Figure 5, from untied RSE simulations of Knudsen and Golubovic (2015), in terms of the center of mass radial distance $r_{c m}(t)$ and angle $\theta_{c m}(t)$ defined in Figure 1(c). The hops appear like a train of "pulses" in $r_{c m}(t)$ separated by time intervals during which the RSE remains nearly at rest with respect to the rotating Earth. During a hop, the RSE top and bottom both rise up and then return back to their initial values while, concurrently, the angle $\theta_{c m}(t)$ undergoes a step-like change, see Figure 5. Between two hops, the RSE bottom rests on the Earth while the $\theta_{c m}$ is nearly constant (see Figure 5), so the RSE is nearly at rest in the geosynchronous frame of the rotating Earth (recall Figure 1(c)). However, the RSE position in this frame changes significantly during the hops during which the RSE bottom displaces from one to another location on the Earth. For example, for the RSE with the gap $D=0.3164$ Earth radii, by Figure 5(b), the RSE bottom's geographical latitude changes by about 1.3 radians during a hop. This is in striking contrast to the simple geosynchronous motion whence the geographical latitude remains constant. See sec. 6 for more discussions of the dumbbell hopping motion.

\section{Multitude of Celestial Dumbbell Modes}

Thus far we elucidated dumbbell slow dynamics within the framework of the simplified dumbbell Lagrangian Equation (9). This model captures dumbbell slow modes but it essentially freezes out fast oscillations of the angles $\theta(t)$ and $\varphi(t)$; see sec. 2 discussions and figs. 1(c) and 3(b). To incorporate these fast dumbbell modes, one must go beyond the model in Equation (9) and consider the exact equations of motion of the celestial dumbbell. Full set of dumbbell equations of motion is obtained by using Lagrangian $L=K E-P E$, with dumbbell kinetic and gravitational potential energy expressed in terms of suitable generalized coordinates, such as the angles $\theta(t)$ and $\theta_{c m}(t)$ (defined in Figure 1(c)), the center of mass radial distance, and the out of plane coordinates $\varphi(t)$ and $\left(R_{2}\right)_{c m}$ (dumbbell tilt tangle and $\mathrm{cm}$ displacement relative to the equatorial plane). By linearizing Euler-Lagrange equations of motion around the dumbbell dynamical equilibrium state Equation (1) one can find the frequencies of all dumbbell modes, as detailed by Knudsen \& Golubovic (2015). They find two in-plane modes (proceeding 
within the Earth equatorial plane), and two out-of-plane modes (proceeding perpendicular to the equatorial plane); see Figure 6. It turns out that, within the linearized theory, two in-plane modes (slow and fast) are decoupled from the two out-of-plane modes (slow and fast). Frequencies of each pair of modes obey biquadratic equations that can be exactly solved. Analytic results for these modes' frequencies are rather cumbersome and transparent physical insight is gained by representing various modes frequencies as expansions in powers of the small parameter $R_{E} / r_{\text {geo }}$ (as we already did in sec. 2 with the frequency of the slow in-plane mode; recall Equation (22)). In this form, the distinction between slow and fast modes becomes completely transparent.

Thus, the solutions of the biquadratic equation for angular frequencies of the in-plane modes are found in the form,

$$
\begin{aligned}
& \left(\frac{\Omega_{\text {slow }}^{\text {in }}}{\Omega_{E}}\right)^{2} \\
& =1-6 \bar{D}+\frac{R_{E}}{r_{\text {geo }}}\left(6 \bar{D}+6 \bar{D}^{2}\right)+\left(\frac{R_{E}}{r_{\text {geo }}}\right)^{2}\left(-12 \bar{D}^{2}-8 \bar{D}^{3}\right)+\left(\frac{R_{E}}{r_{\text {geo }}}\right)^{3}\left(-12 \bar{D}+18 \bar{D}^{2}+20 \bar{D}^{3}+10 \bar{D}^{4}\right)+\ldots
\end{aligned}
$$

for the slow in-plane mode depicted in Figure 6(a); here, as before, $\Omega_{E}=2 \pi / T_{d a y} \quad\left(T_{d a y}=\right.$ sidereal day). On the other hand, for the fast in-plane mode, depicted in Figure 6(b), one finds

$$
\left(\frac{\Omega_{\text {fast }}^{\text {in }}}{\Omega_{m}}\right)^{2}=1+\frac{R_{E}}{r_{\text {geo }}}+\left(\frac{R_{E}}{r_{\text {geo }}}\right)^{2}(1-\bar{D})+\left(\frac{R_{E}}{r_{\text {geo }}}\right)^{3}\left(-2 \bar{D}+\bar{D}^{2}\right)+\ldots,
$$

a)

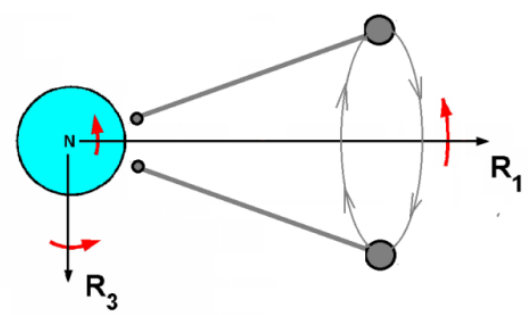

c)

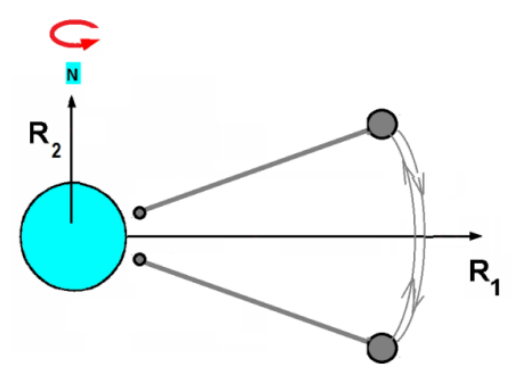

b)

fast in-plane mode

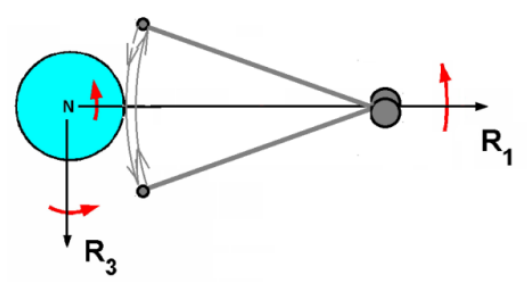

d)

fast out-of-plane mode

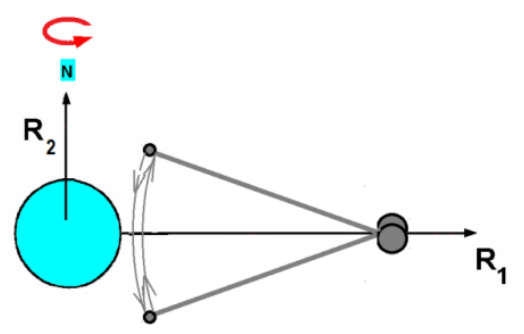

Figure 6. Dumbbell modes: (a) Slow in-equatorial plane mode, best seen in the center of mass coordinates $\theta_{c m}(t) \approx \theta_{M}(t)$ and $r_{c m}(t) \approx r_{M}(t)$; see Figure 1(c). (b) Fast in-equatorial plane mode, best seen in the bottom dynamics or the angle $\theta(t)$ in Figure 1(c). (c) Slow out-of-equatorial plane mode, best seen in the center of mass $R_{2}$ coordinate. (d) Fast out-of-equatorial plane mode, best seen in the bottom $R_{2}$ coordinate or the angle $\varphi(t)$. Adapted from Knudsen \& Golubovic (2015)

with $\Omega_{m}=\left(G M_{E} / R_{E}^{3}\right)^{1 / 2}$, the angular velocity of a satellite in a circular orbit with the radius= $R_{E}$. For $R_{E}=1$ Earth radius; $\Omega_{m}=2 \pi / T_{m}$, where $T_{m}=84.49 \mathrm{~min}$ is the orbital period of the satellite in a circular equatorial orbit with the radius $=R_{E}=1$ Earth radius. It turns out that all terms in the ellipses in Equations (34) and (35) vanish in the limit $\bar{D}=D / R_{E} \rightarrow 0$ (corresponding to the situation with $m / M \rightarrow 0$, see Equation (8)). Thus, in this limit, 


$$
\begin{gathered}
\left(\frac{\Omega_{\text {slow }}^{\text {in }}}{\Omega_{E}}\right)^{2} \rightarrow 1, \\
\left(\frac{\Omega_{\text {fast }}^{\text {in }}}{\Omega_{m}}\right)^{2} \rightarrow 1+\frac{R_{E}}{r_{\text {geo }}}+\left(\frac{R_{E}}{r_{\text {geo }}}\right)^{2} .
\end{gathered}
$$

On the other side, the solutions of the biquadratic equation for the angular frequencies of the out-of-plane modes are found in the form:

$$
\left(\frac{\Omega_{\text {slow }}^{\text {out }}}{\Omega_{E}}\right)^{2}=1, \quad \text { (exact) }
$$

for the slow out-of-plane mode depicted in Figure 6(c). For the fast out-of-plane mode, depicted in Figure 6(d), one finds,

$$
\left(\frac{\Omega_{\text {fast }}^{\text {out }}}{\Omega_{m}}\right)^{2}=1+\frac{R_{E}}{r_{\text {geo }}}+\left(\frac{R_{E}}{r_{\text {geo }}}\right)^{2}(1-\bar{D})+\left(\frac{R_{E}}{r_{\text {geo }}}\right)^{3}\left(1-\bar{D}^{2}\right)+\ldots
$$

It turns out that all terms in the ellipses in Equation (37) vanish in the limit $\bar{D}=D / R_{E} \rightarrow 0$; so in this limit,

$$
\left(\frac{\Omega_{\text {fast }}^{\text {out }}}{\Omega_{m}}\right)^{2} \rightarrow 1+\frac{R_{E}}{r_{\text {geo }}}+\left(\frac{R_{E}}{r_{\text {geo }}}\right)^{2}+\left(\frac{R_{E}}{r_{\text {geo }}}\right)^{3}
$$

The most notable feature of the above results is a separation of time scales. The time periods of both slow modes have time scale $\sim 2 \pi / \Omega_{E}=T_{E}=T_{d a y}=1$ sidereal day. In contrast to this, the time periods of both fast modes have time scale $\sim 2 \pi / \Omega_{m}=T_{m}=84.49 \mathrm{~min}$, satellite period in low Earth orbit. Note that,

$$
\frac{T_{m}}{T_{E}}=\frac{\Omega_{E}}{\Omega_{m}}=\left(\frac{R_{E}}{r_{\text {geo }}}\right)^{3 / 2}=0.05883 \ldots,
$$

so the separation of time scales emerges due to the smallness of the parameter $R_{E} / r_{\text {geo }}$ which obviously plays a fundamental role in our discussions of celestial scale dumbbells.

The slow in-plane mode was already discussed in section 2 within the simplified dumbbell model in Equation (9) which yields this mode frequency as in Equation (22). By comparing Equation (22) with the exact result in Equation (34), we see that the simplified dumbbell model Equation (9) captures this frequency exactly to the second order in $R_{E} / r_{\text {geo }}$. As depicted in Figure 6(a), the in-plane slow mode primarily involves the oscillations of the center mass coordinates polar $\theta_{c m}(t) \approx \theta_{M}(t)$ and $r_{c m}(t) \approx r_{M}(t)$, which are coupled by the conservation law in Equation (10) effectuating an elliptical orbit of the top seen in geosynchronous frame in Figure 6(a). For this mode, the oscillations of the angle $\theta(t)$ [recall Figure 1(c)] are much smaller than the oscillations in $\theta_{c m}(t)$, by a factor $\sim\left(R_{E} / r_{\text {geo }}\right)^{3}$, so the bottom appears as suspended from the slowly oscillating top, as depicted in Figure 6(a). This result actually justifies the validity of the simplified dumbbell model Equation (9). Equation (34) can be used to calculate the $\bar{D}_{\text {hopping }}=D_{\text {hopping }} / R_{E}$ at which the slow mode frequency vanishes. By truncating the expansion (34) to the third order in $R_{E} / r_{\text {geo }}=1 / 6.6108$ one finds $\bar{D}_{\text {hopping }}=D_{\text {hopping }} / R_{E}=0.20003$. For comparison, by using the biquadratic equation result for the slow mode frequency (which is the exact approach), one finds $\bar{D}_{\text {hopping }}=D_{\text {hopping }} / R_{E}=0.20043$, confirming the high accuracy of the expansion forms displayed here. Equation (34) can be iteratively solved to find the $\bar{D}_{\text {hopping }}=D_{\text {hopping }} / R_{E}$ for which $\Omega_{\text {slow }} \rightarrow 0$, with the result having the form of an expansion in powers of the small parameter $R_{E} / r_{\text {geo }}$,

$$
\bar{D}_{\text {hopping }}=\frac{D_{\text {hopping }}}{R_{E}}=\frac{1}{6}+\frac{7}{36} \frac{R_{E}}{r_{\text {geo }}}+\left(\frac{2}{3}\right)^{4}\left(\frac{R_{E}}{r_{\text {geo }}}\right)^{2}-\frac{1}{12}\left(\frac{R_{E}}{r_{\text {geo }}}\right)^{3}+O\left(\frac{R_{E}}{r_{\text {geo }}}\right)^{4} .
$$

Eq. (39) yields $\bar{D}_{\text {hopping }}=D_{\text {hopping }} / R_{E}=0.20031$ for $r_{\text {geo }}=6.6108 R_{E}$ (after truncating the $\left(R_{E} / r_{\text {geo }}\right)^{4}$ term) in very good agreement with the above mentioned exact result $\bar{D}_{\text {hopping }}=D_{\text {hopping }} / R_{E}=0.20043$. Finally, we note 
that, by Equation (39), the corresponding result in Equation (24), derived from the simplified dumbbell model Equation (9), is actually exact to the second order in $R_{E} / r_{g e o}$. Celestial dumbbells also exhibit a slow out-ofequatorial plane mode depicted in Figure 6(c). The time period of this mode is exactly 1 sidereal day long, as stressed in Equation (36). The feature is a consequence of the rotational symmetry of the planet gravitational potential: tilting (out of the plane) dumbbell's circular orbit (in the inertial frame) produces another orbit solving the equations of motion. In the geosynchronous frame, the tilted orbit is seen as an oscillation about the equatorial plane with the period exactly equal one sidereal day. This mode is best seen by looking at the dumbbell center of mass displacement away from equatorial plane; see Figure 3(d) from RSE simulations of Knudsen and Golubovic (2015). Finally, we comment on the two fast dumbbell modes, with frequencies in Equations (35) and (37). These modes are depicted in figs. 6(b) and (d). As seen in these figures, for the fast modes, the dumbbell center of mass and the top are nearly at rest in geosynchronous frame while the dumbbell bottom executes pendular motion. For the fast in-plane mode, the bottom swings along the equator with the frequency as in Equation (35). As depicted in Figure 6(b), this mode is best seen in the dynamics of the angle $\theta(t)$ defined in Figure 1(c). On the other hand, for the fast out-of-plane mode, the bottom swings along the north-south axis with the frequency as in Equation (37). This mode is best seen in the dynamics of the angle $\varphi(t)$ [the dumbbell tilt angle relative to equatorial plane]; see Figure 6(d). By Equations (35) and (37), these two fast pendular modes have nearly the same frequency. The frequency difference appears only in the small terms $\sim\left(R_{E} / r_{\text {geo }}\right)^{3}$. Moreover, these two frequencies change very little (by $-0.23 \%$, for $r_{\text {geo }}=6.6108 R_{E}$ ) as $D$ changes from 0 to $D_{\text {hopping }}$. One can well approximate these two frequencies by their values at $D=0$ displayed in Equations (35') and (37'). Thus one finds, for the time periods of the two fast modes,

$$
T_{\text {fast }}^{\text {out }}(D) \approx T_{\text {fast }}^{\text {in }}(D) \cong T_{\text {fast }}^{\text {in }}(0)=\frac{T_{m}}{\sqrt{1+\frac{R_{E}}{r_{\text {geo }}}+\left(\frac{R_{E}}{r_{\text {geo }}}\right)^{2}}} \cong 78 \mathrm{~min} .
$$

The above analytic results (for the rigid dumbbell model) are in agreement with the simulations results for the fast modes seen in the floppy RSE dynamics of the angles $\theta(t)$ and $\varphi(t)$; see Knudsen and Golubovic (2015). In these simulations, these two angles were indeed found to oscillate with nearly the same time period $\approx 79 \mathrm{~min}$ which does not appreciably change as $D$ changes from 0 to $D_{\text {hopping }}$.

\section{Dumbbell Unbinding Transition Revisited}

We proceed to discuss dumbbell unbinding transition in more detail. The result for the unbinding threshold $D_{\text {unbinding }}$ in Equation (33) was obtained within approximate dumbbell Lagrangian Equation (9) which ignores inertial effects of the dumbbell bottom mass. To assess the role of these effects and derive an exact analytic result for the unbinding transition location, in this section we discuss the unbinding of rigid dumbbells without doing any approximations. The dumbbell obeys conservation of its mechanical (kinetic plus gravitational potential) energy in the inertial frame,

$$
E(t)=K E(t)+P E(t)=\text { const }
$$

In the initial configuration in Figure 3(a), the dumbbell rotates with angular velocity $\Omega_{E}$ around the Earth, so its initial mechanical energy is simply

$$
E_{i n}=K E_{i n}+P E_{i n}=\frac{M R_{M, e q}^{2} \Omega_{E}^{2}}{2}+\frac{m R_{E}^{2} \Omega_{E}^{2}}{2}-\frac{G M_{E} M}{R_{M, e q}}-\frac{G M_{E} m}{R_{E}} .
$$

If the dumbbell unbinds from the Earth to infinity, its final mechanical energy at $t=\infty$ is just its kinetic energy (sum of the center of mass and internal rotational energy),

$$
E_{f i n}=K E_{f i n}=\frac{(M+m) v_{c m, \infty}^{2}}{2}+\frac{I_{c m} \omega_{\infty}^{2}}{2},
$$

where $v_{c m, \infty}$ is the dumbbell's center of mass velocity at infinity while $\omega_{\infty}$ is its angular velocity at infinity (in the inertial frame). In Eq. (43), $I_{c m}$ is the dumbbell's moment of inertia around its center of mass,

$$
I_{c m}=\frac{m M}{M+m} l^{2}=\frac{m M}{M+m}\left(R_{M, e q}-R_{E}\right)^{2} .
$$

To proceed, let us introduce the ratio 


$$
C=\frac{\omega_{\infty}}{\Omega_{E}},
$$

between the final (at infinity) and initial dumbbell's angular velocity (which is equal to the angular velocity of the Earth).

At the unbinding threshold point,

$$
v_{c m, \infty}=0
$$

Thus, by mechanical energy conservation

$$
E_{i n}=E_{f i n}=\frac{I_{c m} \omega_{\infty}^{2}}{2} .
$$

By Equations (42) through (47), and Equation (3), one finds the equation

$$
\frac{M R_{M, e q}^{2}}{2}+\frac{m R_{E}^{2}}{2}-\frac{M r_{g e o}^{3}}{R_{M, e q}}-\frac{m r_{g e o}^{3}}{R_{E}}=\frac{C^{2}}{2} \frac{m M}{M+m}\left(R_{M, e q}-R_{E}\right)^{2},
$$

to be exactly satisfied at the unbinding threshold point. Combining Equation (48) with Equation (5), gives an equation determining the value of $R_{M, e q}=r_{\text {geo }}+D_{\text {unbinding }}$ which can be solved by an expansion in powers of the small parameter $R_{E} / r_{g e o}$. The result for the unbinding threshold has the form,

$$
\frac{D_{\text {unbinding }}}{r_{\text {geo }}}=2^{1 / 3}-1+\frac{1}{3} \frac{R_{E}}{r_{\text {geo }}}+\frac{2^{5 / 3}}{9}\left(\frac{R_{E}}{r_{\text {geo }}}\right)^{2}\left(1+\frac{3}{4} C^{2}\right)+O\left(\frac{R_{E}}{r_{\text {geo }}}\right)^{3},
$$

truncated to the second order in $R_{E} / r_{\text {geo }}$. Note that the exact result in Equation (49) formally reduces to the corresponding result in Equation (33) for $C \rightarrow 0$. The actual $C=\omega_{\infty} / \Omega_{E}$ is however not zero. The $C$ value can be obtained by numerically integrating equations of dumbbell's motion close to the unbinding threshold. This yields,

$$
C=\frac{\omega_{\infty}}{\Omega_{E}}=0.130216+O\left(\frac{R_{E}}{r_{\text {geo }}}\right),
$$

(Knudsen \& Golubovic, 2015). By the form of Equation (50), one can set $C=0.130216$ in the expansion in Equation (49). Physically, the $C$ term in Equation (49), i.e., the difference between Equations (49) and (33) emerges because the approximate dumbbell Lagrangian Equation (9), used to derive Equation (33), neglects the inertial effects due to dumbbell bottom mass $m$. By comparing the exact expansion in Equation (49) with the result in (33), one can see that these bottom inertial effects are in fact small. They appear in the term which is only of the order $\left(R_{E} / r_{g e o}\right)^{2}$, primarily because the ratio of bottom to top mass is small; recall Equations (5) through (8). For example, for the planet Earth with $r_{\text {geo }}=6.6108 R_{E}$, the truncated expansion result in Equation (49) gives $D_{\text {unbinding }}=2.1057 R_{E}$ which is very close to the aforementioned result $D_{\text {unbinding }}=2.1050 R_{E}$ obtained from the truncated expansion in Equation (33) coming from the simplified dumbbell model Equation (9).

To the best of our knowledge, prior to the work of Knudsen \& Golubovic (2015), there were no previous studies rigorously addressing unbinding of celestial size rigid objects. There was however a heuristic argument put forward by Duboshin suggesting that such a phenomenon might in principle happen. Duboshin noted that a body moving in a circular orbit "might have a velocity which would have been hyperbolic had the entire mass been concentrated in at the mass center"; see the chapter 4 of Beletskii (1965) and Figure 27(a) therein. Here, the wording "hyperbolic center of mass velocity" means that the center of mass velocity $v_{c m}$ satisfies the condition

$$
\frac{v_{c m}}{v_{o}}>2^{1 / 2},
$$

where $v_{o}$ is the orbiting velocity of a point mass in a circular orbit with the radius $r_{c m}$, given by the elementary physics expression, 


$$
v_{o}^{2}=\frac{G M_{E}}{r_{c m}}=\frac{\Omega_{E}^{2} r_{g e o}^{3}}{r_{c m}},
$$

where $r_{c m}$ is the radial distance of the object's center of mass. Conceptually, Equation (51) says that if the extended object is "compressed" to a point mass with the velocity $v_{c m}$ then such a point mass would escape to infinity. [Recall that the ratio of the second and first cosmic speed is $2^{1 / 2}$ for any initial radial distance from the planet center.] Though physically appealing, the Duboshin's unbinding condition (51) is only heuristic and it really does not locate the exact unbinding transition point (Knudsen \& Golubovic, 2015). To illustrate this, let us consider the dumbbell in the dynamical equilibrium in Figure 3(a) rotating in a circular orbit with angular velocity $=\Omega_{E}$. For it,

$$
v_{c m}=\Omega_{E} r_{c m} .
$$

By Equations (52) and (53), the Duboshin's condition (51) is equivalent to the condition,

$$
\frac{r_{c m}}{r_{\text {geo }}}>2^{1 / 3} .
$$

Let us compare this to the results in Equations (33) and (49). By noting that $r_{c m}=\left(M R_{M, e q}+m R_{E}\right) /(M+m)$, and by using Equation (5) for the ratio $m / M$, one finds that the Duboshin's condition Equation (54) is equivalent to

$$
D=R_{M, e q}-r_{g e o}>D_{D u b s s h i n}
$$

with

$$
\frac{D_{\text {Duboshin }}}{r_{\text {geo }}}=2^{1 / 3}-1+3 \cdot 2^{1 / 3}\left(2^{1 / 3}-1\right)\left(\frac{R_{E}}{r_{\text {geo }}}\right)^{2}+O\left(\frac{R_{E}}{r_{\text {geo }}}\right)^{3}
$$

By comparing Equation (56) with the result in Equations (33) and (49), it is clear that $D_{\text {Dubssin }}<D_{\text {unbinding }}$, that is, the Duboshin's condition does not exactly locate the position of the unbinding transition. For example, for the planet Earth with $r_{\text {geo }}=6.6108 R_{E}$, the result in Equation (49) gives $D_{\text {unbinding }}=2.1057 R_{E}$, while by Equation (56), one obtains that $D_{\text {Duboshin }}^{\text {geo }}=1.8669 R_{E}$.

This failure of the heuristic Duboshin's condition to accurately locate the dumbbell unbinding transition point comes from the ad hoc assumption that the extended object is "compressed" to a point (center of mass). In particular, such an assumption severely underestimates the significant effects of gravity on the dumbbell's bottom mass in Figure 3(a) which is close to the Earth and far away from the dumbbell center of mass which is above the geostationary orbit at $r_{g e o}=6.6108 R_{E}$. These gravity effects increase the true unbinding threshold from $D_{\text {Dubshin }}=1.8669 R_{E}$ to $D_{\text {unbinding }}=2.1057 R_{E}$ as found by Knudsen \& Golubovic (2015). They confirmed this analytic result by numerical simulations of untied RSE which independently suggest that $D_{\text {unbinding }} \approx 2.1$. Earth radii.

\section{Further Discussions and Conclusion}

In our discussions, for clarity and simplicity we assumed that dumbbell geosynchronous orbit is in the equatorial plane of the planet. This particular choice is however not essential. Because of nearly perfect spherical symmetry of the planet gravitational field, dumbbells with circular geosynchronous orbits tilted with respect the equatorial plane will exhibit essentially the same physics, e.g., the same values of $D_{\text {hopping }} \approx 0.2$ Earth radii and $D_{\text {unbinding }} \approx 2.1$ Earth radii, and the same spectrum of slow and fast modes that can be excited in orbital plane and out of the orbital plane. Whereas no substantially new physics emerges by tilting the orbital plane, the tilting does induce dumbbell bottom motion relative to the rotating planet. Dumbbells with geosynchronous circular orbits tilted with respect the equatorial plane by some angle $\alpha$ have their bottoms tracing a closed path on the Earth shaped like number 8 centered at the equator. This motion has one sidereal day period, with dumbbell bottom geographical longitude oscillating between $+\alpha$ and $-\alpha$. However, if the slow in-plane mode is also excited [see Figure 6(a)], the bottom path will also reflect the presence of oscillations with a period $T_{\text {slow }}^{\text {in }}=2 \pi / \Omega_{\text {slow }}^{\text {in }}$ which is generally longer than one sidereal day; see figure 4(b). In view of this, the bottom path traced on the Earth surface may not be a closed curve. In addition, one may also have a zero-mode excited, corresponding to circular orbits which are not exactly geosynchronous. In the presence of the zero-mode, the dumbbell bottom path will slowly encircle the planet with a period which may be many days long; see Knudsen \& Golubovic (2015). One should also recall that, in addition 
to these slow modes, dumbbell bottom may also exhibit the fast pendular modes with period $T_{\text {fast }} \cong 78$ min, see section 4. Thus, in general, the celestial scale dumbbells execute complex quasi-periodic motion.

In addition to the aforementioned natural dumbbell modes, celestial dumbbell will also exhibit the modes driven by tidal effects of the Moon and the Sun. Their period of 12 hours is not in resonance with dumbbell natural modes periods, so tidal effects act as small perturbations to the dumbbell dynamics discussed in this review. It remains for future studies to assess in more detail these tidal effects, as well as the effects of the planet weak nonsphericity and possible ways of implementing active control of dumbbell motion. Of particular interest would be active control of the aforementioned zero-mode which may be employed to transport the dumbbell around the globe.

We would like to stress the significance of the condition $D<D_{\text {hopping }} \approx 0.2$ Earth radii needed to ensure the stability of circular geosynchronous dumbbell orbits. It is in fact very undesirable to employ hopping dumbbells with $D>D_{\text {hopping }}$. Such dumbbells recurrently closely approach the homoclinic point corresponding to the unstable maximum of the effective potential in figure 4(d). Because of this, such dumbbells may easily start falling towards the center of the Earth, with their bottoms crushing into the Earth surface. An example for this can be seen in figure 5 in which the dumbbell started falling towards the Earth center after performing five hops. Fine details of this unstable dynamics are highly sensitive to initial conditions and are affected by dumbbell fast modes and by internal motions of the dumbbell such as the internal rotation of RSE in Figure 1(a).

In this review we also highlighted the essential instability of dumbbell circular geosynchronous orbits with $D>D_{\text {hopping }}$ Earth radii. Such a celestial scale dumbbell will eventually unbind from the Earth and escape to infinity, i.e., to interplanetary space. This striking behavior of celestial scale objects was qualitatively anticipated before by Duboshin (Beletskii, 1965), while the first rigorous quantitative analysis of this phenomenon came much later in recent work of Knudsen and Golubovic (2015), as discussed in this review.

It should not escape ones attention that various analytic results displayed in this review are given in the form applicable to celestial scale dumbbells deployed around any rotating planet. The Earth was used just as an example but one can apply here discussed results to any planet with $R_{E}$, the planet radius and $r_{g e o}$, the orbit radius of the satellite synchronously rotating with the planet; see Equation (3). For example, for the dwarf planet Ceres, with $R_{E}=476.2 \mathrm{~km}$ one finds $r_{\text {geo }} / R_{E} \approx 2.5$, using available astronomical data (Ceres, 2017). Using this in combination with the equations (39) and (49), one finds that, for Ceres, $D_{\text {hopping }} / R_{E} \approx 0.27$ while $D_{\text {unbinding }} / R_{E} \approx 1.13$. For the Ceres, the low obit period $T_{m} \approx 138 \mathrm{~min}$, so by equation (40), the time period of fast pendular modes is about 110.5 min. From technological point of view, celestial dumbbells such as untied RSEs would be more easily built on dwarf planets in the main asteroid belt such as Ceres, Vesta, Pallas, and Juno. Strings of such dumbbells would have significantly smaller tensile stresses and they would require much less material to be built on dwarf planets than on the Earth. In fact, as noted by Golubovic and Knudsen (2009), the elliptic RSEs based on the Earth have tensile stresses at their string midpoints exceeding the tensile strength of carbon nanotubes which is $60 \mathrm{GPa}$ for single-wall nanotubes and $150 \mathrm{GPa}$ for multi-wall nanotubes (Yu et al., 2000a, Yu et al., 2000b). The situation is however quite different for elliptic RSEs built on dwarf planets as can be shown by using general results for RSE tensile stresses in Golubovic and Knudsen (2009) and Knudsen and Golubovic (2014). For example, for the Ceres, for the elliptic RSE with $b / R_{E}=0.16$ rotating at the climbing threshold RSE period of $19.7 \mathrm{~min}$, one finds that the maximum tensile stress of the string is only about $4 \mathrm{GPa}$, if the RSE is made out of carbon nanotubes. This is more than ten times smaller than the aforementioned tensile strengths of single-wall and multi-wall carbon nanotubes. This strongly suggests that elliptic RSEs and other celestial scale dumbbells may have certain future on dwarf planets of the Solar system.

On theoretical side, it is pleasing to note that simple dumbbell model with a relatively small number of degrees of freedom can be successfully used to understand much more complex physical systems (field theories) having an infinite number of degrees of freedom. Notable example called in this review is the string forming the untied Rotating Space Elevator (Knudsen \& Golubovic, 2015).

In conclusion, physics of celestial scale dumbbells exhibits rich and interesting multitude of phenomena caused by instabilities of dumbbell geosynchronous motion. Presented results are of universal interest for diverse realizations of celestial scale dumbbells such as the Analemma tower suspended from an asteroid orbiting a planet (Clouds AO, 2017) or untied Rotating Space Elevators (Knudsen \& Golubovic, 2015). Whereas Earth based realizations of celestial dumbbells are precluded by finite tensile strength of nowadays available materials, we find that celestial scale dumbbells are actually feasible on dwarf planets of the Solar system.

\section{Acknowledgments}

We thank WVHTC and NASA for the sub-grant WVHTC-W-NASA-IR-07-1367 of the Grant NNX06AE557: Innovative Research for Next Generation Space Exploration. 


\section{References}

Beletskii, V. V. (1965). Motion of an artificial satellite about its center of mass. Moscow, USSR: Nauka. Retrieved July 1, 2017, from https://archive.org/details/nasa_techdoc_19670006100

Ceres (dwarf planet). (2017, July 1). In Wikipedia, the free encyclopedia. Retrieved July 1, 2017, from https://en.wikipedia.org/wiki/Ceres_(dwarf_planet)

Clouds AO. (2017). Analemma Tower. Retrieved July 1, 2017, from http://www.cloudsao.com/ANALEMMATOWER

Edwards, B. C., \& Westling, E. A. (2003). The Space Elevator: A Revolutionary Earth-to-Space Transportation System. USA: BC Edwards.

Fitzgibbons, T. C., Guthrie, M., En-shi Xu, E., Crespi, V. H., Davidowski, S. K., Cody, G. D., ... Badding, J. V. (2014), Benzene-derived carbon nanothreads. Nature Materials, 14, 43-47. http://dx.doi.org/10.1038/nmat4088

Golubović, L., \& Knudsen, S. (2009). Classical and statistical mechanics of celestial scale spinning strings: Rotating space elevators. Europhysics Letters, 86(3), 34001. http://dx.doi.org/10.1209/0295-5075/86/34001

Golubović, L., \& Knudsen, S. (2017). Rotating space elevators: A new venue in space elevator physics. Applied Physics Research, 9(1), 56. http://dx.doi.org/10.5539/apr.v9n1p56

Hughes, P. C. (2012). Spacecraft Attitude Dynamics. Dover Books on Aeronautical Engineering. UK: Dover.

Kardar, M. (2007). Statistical Physics of Fields. Cambridge, UK: Cambridge University Press.

Knudsen, S., \& Golubović, L. (2014). Rotating space elevators: Physics of celestial scale spinning strings. European Physical Journal Plus, 129, 242. http://dx.doi.org/10.1140/epjp/i2014-14242-8

Knudsen, S., \& Golubović, L. (2015). Physics of untied rotating space elevators. European Physical Journal Plus, 130, 243. http://dx.doi.org/10.1140/epjp/i2015-15243-9

Knudsen, S. \& Golubović, L. (2016). Modeling the physics of sliding objects for rotating space elevators. European Physical Journal Plus, 131, 400. http://dx.doi.org/10.1140/epjp/i2016-16400-4

Nelson, D. R. (2002), Defects and Geometry in Condensed Matter Physics. Cambridge, UK: Cambridge University Press.

Nelson, D. R., Piran, T., \& Weinberg, S. (Eds.). (1988). Statistical Mechanics of Membranes and Surfaces. Singapore: World Scientific.

Yu, M. F., Files, B. S., Arepalli, S., \& Ruoff, R. S. (2000a). Tensile Loading of Ropes of Single Wall Carbon Nanotubes and their Mechanical Properties. Physical Review Letters, 84, 5552-5555. http://dx.doi.org/10. 1103/PhysRevLett.84.5552

Yu, M. F., Lourie O., Dyer M. J., Moloni, K., Kelly, T. F., \& Ruoff. R. S. (2000b). Strength and breaking mechanism of multiwalled carbon nanotubes under tensile load, Science, 287, 637-640. http://dx.doi.org/10. $1126 /$ science.287.5453.637

\section{Copyrights}

Copyright for this article is retained by the author(s), with first publication rights granted to the journal.

This is an open-access article distributed under the terms and conditions of the Creative Commons Attribution license (http://creativecommons.org/licenses/by/4.0/). 